\title{
Development of Computerized Adaptive Testing for Emotion Regulation
}

\author{
Lingling X $\mathrm{u}^{1}$, Ruyi Jin', Feifei Huang ${ }^{1}$, Yanhui Zhou' ${ }^{1}$, Zonglong Li' ${ }^{1}$ and \\ Minqiang Zhang ${ }^{1,2,3,4 *}$
}

${ }^{1}$ School of Psychology, South China Normal University, Guangzhou, China, ${ }^{2}$ Key Laboratory of Brain, Cognition and Education Sciences (South China Normal University), Ministry of Education, Guangzhou, China, ${ }^{3}$ Center for Studies of Psychological Application, South China Normal University, Guangzhou, China, ${ }^{4}$ Guangdong Key Laboratory of Mental Health and Cognitive Science, South China Normal University, Guangzhou, China

Emotion regulation (ER) plays a vital role in individuals' well-being and successful functioning. In this study, we attempted to develop a computerized adaptive testing (CAT) to efficiently evaluate ER, namely the CAT-ER. The initial CAT-ER item bank comprised 154 items from six commonly used ER scales, which were completed by 887 participants recruited in China. We conducted unidimensionality testing, item response theory (IRT) model comparison and selection, and IRT item analysis including local

OPEN ACCESS

Edited by:

Laura Badenes-Ribera,

University of Valencia, Spain

Reviewed by:

Nathaniel Haines,

The Ohio State University,

United States

Rudolf Debelak,

University of Zurich, Switzerland

*Correspondence:

Minqiang Zhang

zhangmq1117@qq.com

Specialty section:

This article was submitted to

Quantitative Psychology and Measurement,

a section of the journal

Frontiers in Psychology

Received: 12 May 2020

Accepted: 05 November 2020

Published: 01 December 2020

Citation:

$X u$ L, Jin R, Huang F, Zhou Y, Li Z and Zhang M (2020) Development of Computerized Adaptive Testing

for Emotion Regulation.

Front. Psychol. 11:561358.

doi: 10.3389/fpsyg.2020.561358 independence, item fit, differential item functioning, and item discrimination. Sixty-three items with good psychometric properties were retained in the final CAT-ER. Then, two CAT simulation studies were implemented to assess the CAT-ER, which revealed that the CAT-ER developed in this study performed reasonably well, considering that it greatly lessened the test items and time without losing measurement accuracy.

Keywords: emotion regulation, computerized adaptive testing, item response theory, item bank, measurement

\section{INTRODUCTION}

Emotion regulation (ER) has received substantial and increased attention in psychology and related fields (Campos et al., 2011), as its processes affect an individual's experience of positive and negative emotions, interpersonal relationships, as well as physical health (Gross and John, 2003). ER has long been regarded as a crucial mechanism underlying physical and mental health. Research into ER skills is linked to psychological well-being, social relations, and emotional functioning (Gross and John, 2003; Balzarotti et al., 2010; Gullone and Taffe, 2011). Both theoretical and empirical findings indicate ER's critical role in many areas of psychopathology research, involving anxiety (Mennin et al., 2009), eating (Lavender et al., 2014), and personality disorders (Bornovalova et al., 2008). Therefore, it is important to make an accurate evaluation and diagnosis of those with ER difficulties and offer timely treatment.

Recently, there has been a proliferation of self-report ER measures (Aldao et al., 2010; Megreya et al., 2018), such as the Difficulties in Emotion Regulation Scale (DERS; Gratz and Roemer, 2004), Negative Mood Regulation Scale (NMR; Catanzaro and Mearns, 1990), Regulatory Emotional Self-Efficacy Scale (RESE; Caprara et al., 2008), Emotion Regulation Questionnaire (ERQ; Gross and John, 2003), Trait Meta-Mood Scale (TMMS; Salovey et al., 1995), and Cognitive Emotion Regulation Questionnaire (CERQ; Garnefski et al., 2001). Previous studies (Garnefski et al., 2001; Gross and John, 2003; Gratz and Roemer, 2004; Caprara et al., 2008) suggest that each measure 
mentioned above is formed based on the same underlying structure (i.e., ER). Example items of these measure can be seen in the "Measures" section. The description based on the content of the individual items may aid in better reflecting the same structure of these measures to a certain extent. Additionally, critical evidence from several meta-analytic reviews indicates each scale measures and evaluates the same underlying ER structure. For example, Naragon-Gainey et al. (2017) showed that self-report ER measures, including the ERQ, DERS, and CERQ, have been used to examine the underlying structure of common ER and evaluate it in light of the theoretical models of ER. Aldao et al.'s (2010) study also found that the aforementioned self-report scales were used to measure and evaluate ER. Furthermore, several recent studies reported that the DERS, ERQ, RESE, CERQ, NMR, and TMMS have been incorporated into a common structural model of ER (Gross, 2014; Megreya et al., 2018).

To date, these self-report ER measures have only been applied using the paper-and-pencil $(\mathrm{P} \& \mathrm{P})$ method. $\mathrm{P} \& \mathrm{P}$ questionnaires are usually onerous for the subjects, because all items need to be administered regardless of whether they are related or not. For instance, certain items may be highly difficult or extremely easy for the individual, relying on their level of the construct being measured (e.g., ER). Meanwhile, the collection of subjects' responses employing $\mathrm{P} \& \mathrm{P}$ measures demands subsequent data entry and scoring calculations, both of which warrant considerable resources and expertise.

One way to address the abovementioned problems is by performing adaptive testing. Computerized adaptive testing (CAT) typically involves an item-level adaptive test, a form of testing that employs item response theory (IRT) to build an item bank, and automatically chooses appropriate items from it according to the subject's ability level, which is updated based on the subject's responses to each item. This process continues until the test taker's theta reaches satisfactory accuracy. In contrast to $\mathrm{P} \& \mathrm{P}$ testing, the primary advantage of CAT is that it demands fewer test items and takes less time to achieve similarly accurate scores, lessening administration burden and ensuring subjects' motivation. Additionally, the test developer can preset the degree of measurement accuracy demanded in a CAT design. More importantly, CAT automates the administration of data, scoring, and reporting, which enables the results to be integrated into feedback and treatment immediately.

Given these benefits, CAT and item banking are becoming increasingly popular in the field of ability measurement and psychology, including some large-scale tests such as the Graduate Record Examination (GRE) and Test of English as a Foreign Language (TOEFL), as well as tests to assess anxiety (Walter et al., 2007) and depression (Gardner et al., 2004). Considering the drawbacks of P\&P testing and the advantages of CAT, this study attempted to offer a novel ER evaluation technique by employing CAT as the measurement method with a Chinese sample. More specifically, this study addressed some existing critical issues. First, a calibrated item bank with good psychometric properties was developed in this study. Second, the reliability and validity of the CAT-ER were assessed using two simulation studies in different stopping rules. Third, we provide recommendations for applicators who plan to put adaptive testing into use.

\section{MATERIALS AND METHODS}

\section{Participants}

A total of 887 Chinese adolescents and adults living in 29 randomly selected cities in China, aged between 16 and 64 years (median $=30.612$ years, $S D=13.527$ ), participated in this study (83.3\% response rate). Respondents were recruited from July to September 2019. Table 1 contains detailed demographic characteristics. The sample included 549 females (61.9\%) and 338 males $(38.1 \%)$. There were 448 (50.5\%) participants under 25 years old and $439(49.5 \%)$ aged 25 and above. The participants came from urban $(55.1 \%)$ and rural $(44.9 \%)$ regions. Volunteers anonymously completed the demographic questions and selfreport measures online. The purpose of this study, assurances of confidentiality, and participants' rights were explained to them. All subjects provided written informed consent and were paid for their participation. The current study was approved by the local Ethics Committee of the School of Psychology, South China Normal University.

\section{Measures}

Drawing on the findings from prior studies, the 154 items from six frequently employed Chinese-versions of self-report ER measures were used to build the initial CAT-ER item bank (Table 2). The ER measures used in this study included the DERS (Li and Wu, 2018), TMMS (Li et al., 2002), RESE (Wen et al., 2009), ERQ (Li and Wu, 2018), CERQ (Zhu et al., 2008), and NMR (Wang et al., 2017). Example items of the DERS are "I am clear about my feelings" and "When I'm upset, I believe my emotions are valid and important." Example items of the TMMS are "I try to think of good thought no matter how badly I feel" and "No matter how badly I feel, I try to think about pleasant things." Example items of the RESE are "Express enjoyment freely at parties" and "Avoid flying off the handle when you get angry." Example items of the ERQ are "I keep my emotions to myself" and "I control my emotions by not expressing them." Example items of the CERQ are "I think that I have to accept the situation" and "I think about how to change the situation." Example items of the NMR are "I can do something to feel better" and "I can find a way to relax." Each of the five measures (i.e., DERS, TMMS, RESE, CERQ, NMR) is scored on a 5-point Likert scale, and the ERQ is scored on a 7-point Likert scale. Higher scores for several items of the DERS, NMR, and RESE indicate greater difficulties in ER. In this study, we attempted to measure the ability of individual successful ER, reverse-coded items thus needed to be forward coded. Through the above approach, a higher total score of these measures denoted a more successful ER performance.

We implemented two types of analyses to develop the CATER based on the IRT. The first analysis was to satisfy the psychometric requirements of the CAT-ER, and the second was to simulate the CAT adaptively using existing item responses and simulated responses. Friedman et al. (2010) stated that overfitting and overly optimistic results might appear when 
TABLE 1 | Demographic characteristics $(N=887)$.

\begin{tabular}{lccc}
\hline Variables & Category & Frequency & Percent (\%) \\
\hline Gender & Male & 338 & 38.1 \\
& Female & 549 & 61.9 \\
\multirow{2}{*}{ Age } & Under 25 years & 448 & 50.5 \\
\multirow{2}{*}{ Region } & 25 and above & 439 & 49.5 \\
& Rural & 398 & 44.9 \\
& Urban & 489 & 55.1 \\
\hline
\end{tabular}

the same sample is used to estimate item parameters and simulate CAT studies. Therefore, the real 887 subjects' responses collected in advance were randomly divided into a training and a test set. Reise and Yu (1990) suggested, a sample size of at least 500 is considered enough for acceptable item parameter estimates for unidimensional polytomous IRT models through simulation studies in the item bank calibration. Therefore, we applied the real 500 subjects' responses in constructing the CATER item bank and used the real 387 subjects' responses to simulate the CAT-ER to confirm the precision and validity of the algorithm. Furthermore, to test the performance of this method on the test set after iterating all item reduction methods on the training set, the real 387 subjects' responses also were used to validate the psychometric properties of the final item set of the CAT-ER.

\section{Construction of CAT-ER Item Bank Unidimensionality}

In IRT, unidimensionality is an important assumption, and an item bank is deemed unidimensional when a subject's item responses derives from the subject's actual trait level that the item measures and not from other elements. Therefore, there is a need to assess the unidimensionality of the item bank for IRT applications (Reise et al., 2007). A factor analytical framework and an IRT framework were used to evaluate unidimensionality.

First, data were randomly divided into two sets $(n=250$ each), one for exploratory factor analysis (EFA) and the other for confirmatory factor analysis (CFA). In EFA, we used parallel analysis as the criterion to estimate the number of meaningful factors. Items with factor loadings over 0.30 that were significant at $p=0.05$ were considered for retention in the development of the item bank. In CFA, the root mean square error of approximation (RMSEA), comparative fit index (CFI), and Tucker-Lewis index (TLI) were utilized to evaluate the goodness of fit. Researchers have pointed out that RMSEA $\leq 0.08$, CFI $\geq 0.90$, and TLI $\geq 0.90$ denote an acceptable fit (Kline, 2010). Next, we calculated the proportion of total variance attributable to a general factor with omega hierarchical $\left(\omega_{h}\right) . \omega_{h} \geq 0.70$, indicating that the item set is fully unidimensional (Reise et al., 2013). Moreover, the proportion of explained common variance (ECV) is also a useful index for determining the importance of the general factor. The ECV cut-off value in a bifactor model is 60\%, and a higher value means better performance (Reise et al., 2013).

Additionally, unidimensionality was examined by a descriptive analysis of the standardized residuals of item responses for the IRT model. A goodness-of-fit test was conducted from a fitted IRT model to detect violations of the unidimensionality hypothesis in the test data (Finch and Habing, 2007). The $M_{2}$ statistic was employed to test the overall goodness of fit of a parametric model (Maydeu-Olivares and Joe, 2006).

\section{IRT Model Selection}

In the IRT framework, a suitable model is needed for parameter estimation. Some frequently employed multi-index IRT models for polytomous data involve the graded response model (GRM; Samejima, 1969), the generalized partial credit model (GPCM; Muraki, 1992), and the nominal response model (NRM; Bock, 1972). We chose an optimal model for further analysis based on the following test-level model fitting indexes: -2 loglikelihood (-2LL; Spiegelhalter et al., 1998), Akaike’s information criteria (AIC; Akaike, 1974), Bayesian information criterion (BIC; Schwarz, 1978), and $M_{2}$ statistic (Maydeu-Olivares and Joe, 2006). Smaller values of these indexes reflect a better model fit. The goodness of fit ( $M_{2}$ statistic) was not statistically significant, indicating that the data fit well in the model.

\section{Local Independence}

To ensure that parameter estimates are not excessively distorted due to related item pairs, we evaluated local dependence in the IRT framework with residual correlations and Yen's (1993) Q3 statistic. Based on a prior study (Flens et al., 2017), residual correlation values of 0.20 or greater and Yen's Q3 statistic values of 0.36 or higher were deemed large and unexpected.

TABLE 2 | Items from six scales.

\begin{tabular}{|c|c|c|}
\hline Scale & Number of items & Items \\
\hline DERS & 36 & $\begin{array}{l}\text { DERS-1, 2, 3, 4, 5, 6, 7, 8, 9, 10, 11, 12, 13, 14, 15, 16, 17, 18, 19, 20, 21, 22, 23, 24, 25, 26, 27, 28, 29, 30, 31, 32, 33, } \\
34,35 \text {, and DERS-36 }\end{array}$ \\
\hline TMMS & 30 & TMMS-1, 2, 3, 4, 5, 6, 7, 8, 9, 10, 11, 12, 13, 14, 15, 16, 17, 18, 19, 20, 21, 22, 23, 24, 25, 26, 27, 28, 29, and TMMS-30 \\
\hline RESE & 12 & RESE-1, 2, 3, 4, 5, 6, 7, 8, 9, 10, 11, and RESE-12 \\
\hline $\mathrm{ERQ}$ & 10 & $\mathrm{ERQ}-1,2,3,4,5,6,7,8,9$, and $\mathrm{ERQ}-10$ \\
\hline CERQ & 36 & $\begin{array}{l}\text { CERQ-1, 2, 3, 4, 5, 6, 7, 8, 9, 10, 11, 12, 13, 14, 15, 16, 17, 18, 19, 20, 21, 22, 23, 24, 25, 26, 27, 28, 29, 30, 31, 32, 33, } \\
34,35 \text {, and CERQ-36 }\end{array}$ \\
\hline NMR & 30 & NMR-1, 2, 3, 4, 5, 6, 7, 8, 9, 10, 11, 12, 13, 14, 15, 16, 17, 18, 19, 20, 21, 22, 23, 24, 25, 26, 27, 28, 29, and NMR-30 \\
\hline
\end{tabular}

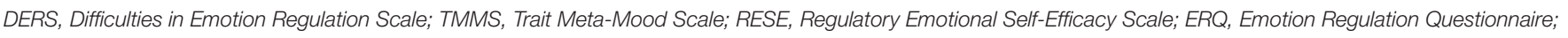
$C E R Q$, Cognitive Emotion Regulation Questionnaire; NMR, Negative Mood Regulation Scale. 


\section{Item Fit}

Item fit was investigated using the $S-\chi^{2}$ statistic (Kang and Chen, 2008). Items with a $S-\chi^{2} p<0.01$ were considered to have a poor fit and were thus removed from the item bank.

\section{Differential Item Functioning}

Ordinal logistic regression (Crane et al., 2006) was applied to assess Differential Item Functioning (DIF) using the R package "lordif" (Choi, 2015). Change in McFadden's pseudo- $R^{2}$ was employed to assess the effect size, and the hypothesis of no DIF was rejected when $\Delta R^{2}>0.02$ and $p>0.05$ (Flens et al., 2017), and such items were excluded. We assessed DIF for gender, age, and region groups.

\section{Item Discrimination}

Researchers suggest that a discrimination value of $0.50-2.50$ indicates an acceptable IRT discrimination (Chang and Ying, 1996). In this study, items with poor discrimination values (i.e., discrimination value $<0.50$ ) were removed from the item bank.

Based on the steps detailed above, all items that satisfied the psychometric requirements were retained to develop the final item set of the CAT-ER. To test the performance of this method on the test set after iterating all item reduction methods on the training set, a similar evaluation (i.e., unidimensionality, local independence, item fit and DIF) was conducted on the test set. We used the R package "psych" for EFA and bifactor analysis, and Mplus 7.0 (Muthén and Muthén, 2012) for CFA. The IRT analyses (involving unidimensionality, model selection, local independence, item fit, and item discrimination) were performed using the R package "mirt" (Chalmers, 2012).

\section{Simulation of the CAT-ER}

The CAT simulation was implemented after the final item set of the CAT-ER was built. Based on the real-item parameters of the CAT-ER, the performance of the CAT-ER using simulated data in various ER levels was simulated to assess its rationality. The abilities of 1,000 virtual persons were generated in random from $N(0,1)$; this sample was considered to represent the true theta values. The advantage of simulating new thetas includes the ability to reach full ranges of the subjects with various theta values.

There were four stages in the CAT simulation, including the initial, test, stop, and final stages (Magis and Raiche, 2012). In the initial stage, the first item was chosen randomly from the final item set of the CAT-ER; then, the subject's response was simulated based on the true theta value simulated before and the randomly chosen initial item. Furthermore, the theta value was estimated using the expected a posteriori method (EAP; Bock and Mislevy, 1982) according to their responses and item parameters. In the test stage, the Fisher information values for each remaining item were calculated. Then, at the temporary estimate of the new theta, the next item was chosen based on the maximum Fisher information criterion (Linden, 1998), which is one of the most frequently employed criteria for item selection in CAT. In the stop stage, CAT stopped when the standard error (SE) of theta achieved $0.447 / 0.386 / 0.316 / 0.224$, which represented measurement reliabilities of $0.800 / 0.850 / 0.900 / 0.950$, respectively. The final stage presented all the analysis results, including the final estimated theta, number of response items, and standard error of measurement (SEM). We used the R package "catR" (Magis and Barrada, 2017) for the analysis.

Furthermore, a real-data simulation of the CAT-ER was also conducted to check the quality of the item bank thoroughly. There is a difference between the real-data CAT-ER simulation and the simulated-data CAT-ER simulation. The former used the real 387 subjects' responses collected before, whereas the latter employed the simulated subjects' responses. Item parameters and the real 387 subjects' responses were input by employing the realdata CAT-ER simulation. The CAT also stopped when the SE $(\theta)$ was $0.447 / 0.386 / 0.316 / 0.224$.

In CAT, efficiency and reliability are important factors for performance quality. Marginal reliability is easy to use and dynamically monitors the reliability of CAT (Green et al., 1984). Generally, marginal reliability refers to a function of SEM, as illustrated in Eqs. 1, 2. The greater the marginal reliability was, the smaller the SEM was. Moreover, marginal reliability is equal to the average reliability for all participants (Wainer et al., 2000) and calculated as follows:

$$
\begin{gathered}
M R=1-S E^{2} \\
S E=\frac{\sum_{i=1}^{N} S E\left(\theta_{i}\right)}{N}
\end{gathered}
$$

In Eq. 2, $N$ is the total number of examinees, $i$ is the specific subjects, and $\operatorname{SE}\left(\theta_{i}\right)$ is the standard error of subject $i$ at the final estimated $\theta$. In addition, marginal reliability increases with decreasing SE. In this article, several statistics were conducted to investigate the efficiency and reliability of CAT-ER, including the mean and standard deviation of the selected items, mean $\mathrm{SE}$, marginal reliability, the mean and standard deviation of estimated theta, and Pearson's correlations between the estimated theta with the stopping rule of None and the remnant stopping rules. The number of selected items with the marginal reliability for each subject was plotted under several stopping rules employing the R package "ggplot2" (Wickham, 2011).

Validity is another essential index of performance quality in CAT. Only when the evaluation result of the CAT-ER is similar to that of the calibration scale, CAT-ER can be considered effective. For that purpose, we used calibration-related validity to evaluate the similarity. The revised Life Orientation Test (LOTR) was chosen as the calibration scale, as it has been widely used to assess optimism (Scheier and Carver, 1985; Scheier et al., 1995). Furthermore, we employed the Chinese version of LOT-R translated by Gu and Wang (2012) to implement the analysis of criterion-related validity of CAT-ER. LOT-R has 10 items, including four filling items, three positive items, and three negative items, all scored on a 5-point Likert-type scale. The total score was the general index of optimism tendency, while the positive and negative item scores were used to measure optimism and pessimism, respectively. The criterionrelated validity of CAT-ER was assessed using SPSS version 23 (George and Mallery, 2016). 


\section{RESULTS}

\section{Item Bank Construction of CAT-ER Unidimensionality}

Forty-five items were removed in the EFA since the factor loadings were $<0.30$ or not significant $(p>0.05)$. After excluding 45 items with low factor loadings or non-significance from the item bank, we ran the one-factor model CFA based on the remaining 109 items. Findings indicated an acceptable model fit in CAF (RMSEA $=0.088, \mathrm{CFI}=0.955$, and TLI $=0.945$ ). The findings suggested that the remaining 109 items (see Table 4) were sufficiently unidimensional. Consistent with these findings, the generated a high value (0.874) and the ECV also had a high value $(65.4 \%)$ indicating the existence of a dominant general ER factor. For the remaining items, the GRM fit the data well, which is evidenced by absolute values of standardized residuals $<1.96$ for all items. The goodness of fit was not statistically significant $\left(M_{2}=7740.187, d f=496, p=0.070\right)$, suggesting that the 109 items indicated a single construct.

\section{IRT Model Selection}

Of the three models, the GRM fitted the remaining items best, as it had the smallest $-2 \mathrm{LL}$, AIC, and BIC values, and a nonsignificant goodness of fit $\left(M_{2}=7740.187, d f=496, p=0.070\right)$ (Table 3). Thus, the GRM was employed to analyze the final CAT-ER item bank.

TABLE 3 | Model-fit indices.

\begin{tabular}{lcccc}
\hline Model & $-\mathbf{2 L L}$ & AIC & BIC & $\mathbf{M}_{\mathbf{2}}$ \\
\hline Graded Response Model & $240,265.2$ & $241,375.2$ & $244,032.5$ & $7,740.187$ \\
Generalized Partial Credit & $243,782.4$ & $244,892.5$ & $247,549.5$ & $7,936.263$ \\
Model & & & & \\
Nominal Response Model & $241,316.8$ & $243,100.8$ & $247,371.6$ & $7,779.689$
\end{tabular}

-2LL, -2Log-Likelihood; AIC, Akaike's information criterion; BIC, Bayesian information criterion; $M_{2}$ statistic is one of the widely used indices to test the overall goodness of fit.

TABLE 4 | Reasons for stepwise exclusion of the items.

\begin{tabular}{ll}
\hline Excluded reasons & Excluded items \\
\hline Unidimensionality & DERS-3, 5, 6, 11, 17, 18, 20, 21, 23, 32, 33, and 34 \\
& TMMS-2, 8, 15, 20, 22, 25, 26, 27, and 28 \\
& RESE-3 and 8 \\
& ERQ-2, 4, 6, 8, and 9 \\
& CERQ-3, 4, 6, and 16 \\
& NMR-2, 7, 9, 10, 11, 12, 13, 19, 22, 25, 26, 28, and 30 \\
& DERS-2, 7, 16, 22, 28, 29, 35, and 36 \\
Local Independency & TMMS-5, 12, 13, 14, 18, and 19 \\
& RESE-9, 10, 11, and 12 \\
& ERQ-10 \\
& CERQ-9, 10, 11, 12, 13, and 14 \\
& NMR-1 and 3 \\
S- $x^{2}$ & CERQ-19, 20, 21, 22, 23, 24, 25, 26, 27, 28, 29, 30, \\
& $31,32,33,34,35$, and 36 \\
DIF & NMR-5 \\
Discrimination & None \\
& None
\end{tabular}

TABLE 5 | Item parameters for 63-item bank with GRM.

\begin{tabular}{|c|c|c|c|c|c|c|c|}
\hline Item & a1 & b1 & b2 & b3 & b4 & b5 & b6 \\
\hline DERS-1 & 1.294 & -3.020 & -1.016 & 0.018 & 1.917 & & \\
\hline DERS-4 & 0.943 & -5.102 & -3.054 & -1.066 & 1.650 & & \\
\hline DERS-8 & 1.243 & -4.018 & -1.667 & -0.273 & 1.858 & & \\
\hline DERS-9 & 0.971 & -2.932 & -0.738 & 1.179 & 4.294 & & \\
\hline DERS-10 & 1.250 & -3.649 & -1.734 & -0.108 & 1.979 & & \\
\hline DERS-12 & 1.606 & -1.987 & -1.251 & 0.154 & 1.343 & & \\
\hline DERS-13 & 0.771 & -4.229 & -2.116 & -0.300 & 2.608 & & \\
\hline DERS-14 & 0.977 & -3.166 & -1.064 & 1.024 & 3.151 & & \\
\hline DERS-15 & 0.842 & -3.609 & -2.054 & 0.804 & 3.180 & & \\
\hline DERS-19 & 0.642 & -4.982 & -1.985 & 0.980 & 3.364 & & \\
\hline DERS-24 & 1.336 & -2.791 & -0.924 & 0.558 & 2.088 & & \\
\hline DERS-25 & 0.898 & -4.137 & -1.353 & 0.892 & 3.389 & & \\
\hline DERS-26 & 1.150 & -3.242 & -1.331 & 0.651 & 1.944 & & \\
\hline DERS-27 & 1.582 & -2.558 & -1.015 & 0.412 & 1.329 & & \\
\hline DERS-30 & 1.424 & -2.092 & -1.022 & 0.121 & 1.310 & & \\
\hline DERS-31 & 1.406 & -2.047 & -1.325 & -0.206 & 1.318 & & \\
\hline TMMS-1 & 1.363 & -3.201 & -1.559 & -0.075 & 1.527 & & \\
\hline TMMS-3 & 1.019 & -2.581 & -0.683 & 0.675 & 2.394 & & \\
\hline TMMS-4 & 1.782 & -2.056 & -0.838 & 0.084 & 1.175 & & \\
\hline TMMS-6 & 0.881 & -3.358 & -1.512 & 0.324 & 1.609 & & \\
\hline TMMS-7 & 0.786 & -3.704 & -1.791 & 0.340 & 1.809 & & \\
\hline TMMS-9 & 0.989 & -2.789 & -1.339 & 0.380 & 1.470 & & \\
\hline TMMS-10 & 0.823 & -3.297 & -1.433 & 0.686 & 2.365 & & \\
\hline TMMS-11 & 1.086 & -3.047 & -0.938 & 0.583 & 2.642 & & \\
\hline TMMS-16 & 1.166 & -2.979 & -0.695 & 0.823 & 3.121 & & \\
\hline TMMS-17 & 0.926 & -4.258 & -1.766 & 0.142 & 2.249 & & \\
\hline TMMS-21 & 1.212 & -3.655 & -1.699 & -0.181 & 2.141 & & \\
\hline TMMS-23 & 1.518 & -2.949 & -1.153 & 0.029 & 1.841 & & \\
\hline TMMS-24 & 1.094 & -2.865 & -1.163 & 0.306 & 2.719 & & \\
\hline TMMS-29 & 1.465 & -2.654 & -0.817 & 0.319 & 1.848 & & \\
\hline TMMS-30 & 1.410 & -2.615 & -1.251 & 0.265 & 2.534 & & \\
\hline RESE-1 & 1.181 & -4.814 & -2.626 & -0.846 & 1.382 & & \\
\hline RESE-2 & 1.668 & -2.056 & -1.298 & -0.334 & 1.103 & & \\
\hline RESE-4 & 1.907 & -1.473 & -0.672 & 0.210 & 0.925 & & \\
\hline RESE-5 & 1.813 & -1.348 & -0.244 & 0.363 & 0.828 & & \\
\hline RESE-6 & 1.967 & -1.421 & -0.195 & 0.438 & 1.053 & & \\
\hline RESE-7 & 2.318 & -1.262 & -0.265 & 0.323 & 1.035 & & \\
\hline ERQ-1 & 1.968 & -2.802 & -1.735 & -0.820 & 0.042 & 0.861 & 2.071 \\
\hline ERQ-3 & 1.449 & -3.979 & -2.590 & -1.367 & -0.320 & 0.691 & 2.205 \\
\hline ERQ-5 & 1.469 & -3.126 & -1.813 & -0.992 & -0.085 & 0.883 & 2.043 \\
\hline ERQ-7 & 1.114 & -4.805 & -3.180 & -2.030 & -0.457 & 1.092 & 3.316 \\
\hline CERQ-1 & 1.212 & -1.993 & -0.539 & 1.155 & 3.135 & & \\
\hline CERQ-2 & 1.401 & -3.147 & -1.584 & 0.079 & 2.011 & & \\
\hline CERQ-5 & 1.137 & -4.570 & -2.636 & -0.363 & 2.064 & & \\
\hline CERQ-7 & 1.381 & -2.222 & -0.428 & 0.883 & 2.391 & & \\
\hline CERQ-8 & 1.258 & -4.348 & -1.963 & -0.078 & 2.603 & & \\
\hline CERQ-15 & 2.220 & -1.397 & -0.442 & 0.274 & 0.992 & & \\
\hline CERQ-17 & 1.206 & -2.812 & -1.414 & -0.096 & 1.415 & & \\
\hline CERQ-18 & 1.392 & -2.750 & -1.561 & -0.343 & 1.257 & & \\
\hline NMR-4 & 1.018 & -4.053 & -1.961 & -0.221 & 2.641 & & \\
\hline NMR-6 & 1.797 & -2.233 & -1.235 & -0.261 & 1.509 & & \\
\hline NMR-8 & 1.528 & -1.886 & -0.580 & 0.565 & 1.886 & & \\
\hline NMR-14 & 0.968 & -3.312 & -0.775 & 0.948 & 3.457 & & \\
\hline
\end{tabular}

(Continued) 
TABLE 5 | Continued

\begin{tabular}{|c|c|c|c|c|c|c|c|}
\hline Item & a1 & b1 & b2 & b3 & b4 & b5 & b6 \\
\hline NMR-15 & 1.420 & -3.008 & -1.386 & -0.085 & 2.226 & & \\
\hline NMR-16 & 1.624 & -1.922 & -0.485 & 0.638 & 1.646 & & \\
\hline NMR-17 & 1.645 & -2.019 & -1.004 & 0.219 & 1.417 & & \\
\hline NMR-18 & 1.057 & -3.383 & -0.933 & 0.778 & 3.309 & & \\
\hline NMR-20 & 0.972 & -4.725 & -1.871 & 0.267 & 3.473 & & \\
\hline NMR-21 & 0.513 & -6.190 & -0.821 & 2.610 & 7.032 & & \\
\hline NMR-23 & 1.031 & -3.981 & -1.651 & 0.052 & 2.567 & & \\
\hline NMR-24 & 1.383 & -2.128 & -0.497 & 0.825 & 1.988 & & \\
\hline NMR-27 & 1.606 & -1.846 & -0.328 & 0.847 & 1.958 & & \\
\hline NMR-29 & 1.037 & -4.010 & -1.811 & -0.014 & 2.874 & & \\
\hline
\end{tabular}

a, discrimination parameter; $b$, difficulty parameter.

\section{Local Independence}

A total of 29 pairs of items indicated local dependence based on their residual correlations $>0.20$ and Q3 values $>0.36$. One item has local dependence in three item pairs, and we removed this item in the three item pairs. Accordingly, 27 items were eliminated on account of local dependence (Table 4). The remaining items satisfied the local independence well.

\section{Item Fit}

Nineteen items had a poor fit to the GRM $\left(S-\chi^{2}<0.01\right)$ (Table 4). After excluding these items, the remaining 63 items were reevaluated and showed a good fit $\left(S-\chi^{2}>0.01\right)$.

\section{Differential Item Functioning}

None of items' $\Delta R^{2}$ values were higher than 0.02 with corresponding $p$ values higher than 0.05 . Therefore, there was no DIF according to gender, age, or region for any items (Table 4).

\section{Item Discrimination}

A GRM was employed again to calibrate the remaining 63 items. The discrimination parameters of the remaining 63 items were all $>0.50$ with mean of $1.294(S D=0.371)$, indicating a high-quality item bank (Table 5).

The above steps produced a 63-item set of CAT-ER (Table 5) that satisfied the unidimensionality and local independence hypotheses in IRT, fitted the GRM well, had high item discrimination parameters, good item fit, and no DIF. Furthermore, the real 387 subjects' responses were used to validate the psychometric properties of the final item set of the CAT-ER. According to the one-factor CFA model of the remaining 63-item set, acceptable fit indices were obtained $(\mathrm{RMSEA}=0.077, \mathrm{CFI}=0.902$, and TLI $=0.913)$. In addition, the general ER factor of the bifactor model for all items accounted for $78.5 \%$ of the variance in the summed score and $63.5 \%$ of the common variance for all items. Thus, the common variance for all items mainly emerged from the general ER factor. These findings show that the final item set of the CAT-ER fully met the hypothesis of unidimensionality. In addition, the goodness of fit was not statistically significant $\left(M_{2}=5970.956, d f=693\right.$, $p=0.125$ ), indicating that the final item set of the CAT-ER represented a single construct. Furthermore, none of the 63 items indicated local dependence (residual correlations: $<0.20$; Q3 values: $<0.36)$ and the item fit evaluation suggest that all them fitted the GRM $\left(S-\chi^{2}>0.01\right)$. None of the 63 items' $\Delta R^{2}$ values exceeded 0.02 , and the corresponding $p$ values were all $>0.05$ in any of the DIF comparisons (gender, age, and region). Based on these analyses using the real 387 subjects' responses, we confirmed that the items retained in the final CAT-ER item bank have acceptable item characteristics. This also increases the generalizability of study findings to replication studies using a smaller sample.

\section{Simulation of CAT-ER}

\section{Results Based on the Simulated Data of the CAT-ER}

Table 6 shows the simulated data of the CAT-ER findings under different stopping rules. For these stopping rules, the mean number of selected items ranged from 5.135 to 26.079 or 8.2 to $41.4 \%$ of the full-item bank. These findings were satisfactory, particularly with the SE (theta) $\leq 0.447$, the CATER employed merely approximately five items while reaching the full-item efficiency. Moreover, with the SE (theta) $\leq 0.224$, the CAT-ER saved more than half of the item bank usage. The mean SE of theta for several stopping rules ranged from 0.222 to 0.422 . It reveals that corresponding measurement precision was achieved in each stopping rule. Furthermore, the marginal reliabilities under several stopping rules varied from 0.822 to 0.969 , which were mostly acceptable for individuals. Table 6 also presents the Pearson's correlations ( $r$ ) between the CAT-ER theta estimates and full-item bank theta estimates under several stopping rules, which were all greater than 0.900 .

TABLE 6 | Simulated-data simulation statistics for CAT-ER under several stopping rules.

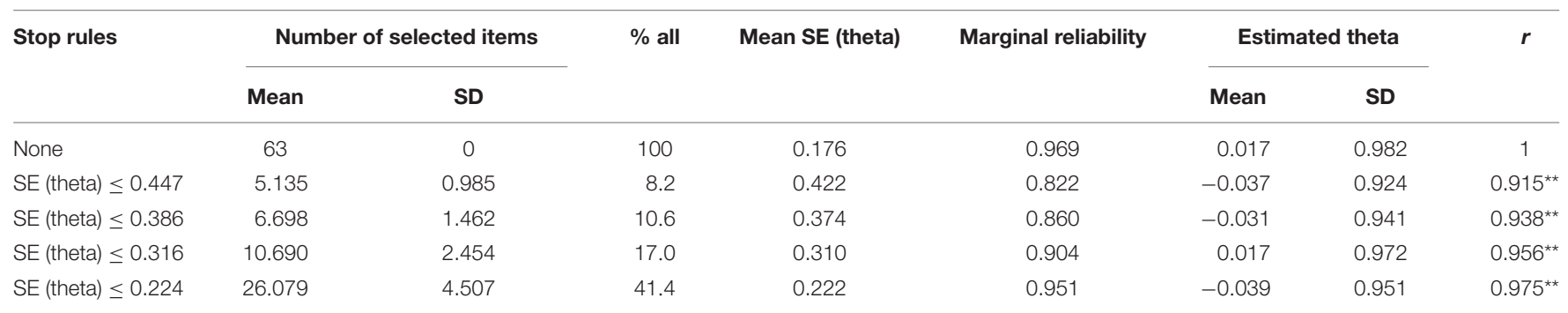

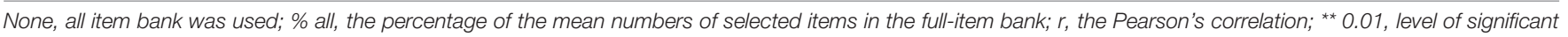
correlation (two-tailed). 


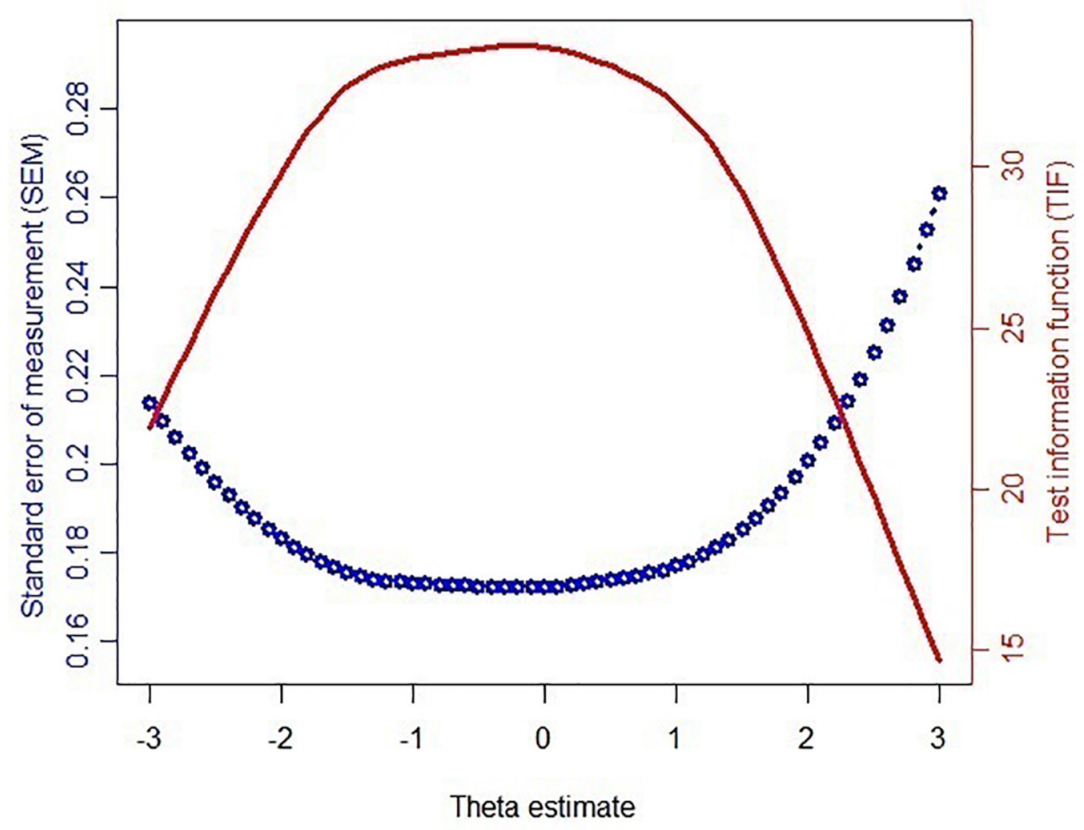

FIGURE 1 | Test information function (TIF) and standard error of measurement (SEM) of the theta values estimated via 63 items of the bank.

TABLE 7 | Real-data simulation statistics for CAT-ER under several stopping rules.

\begin{tabular}{|c|c|c|c|c|c|c|c|c|}
\hline \multirow[t]{2}{*}{ Stop rules } & \multicolumn{2}{|c|}{ Number of selected items } & \multirow[t]{2}{*}{$\%$ all } & \multirow[t]{2}{*}{ Mean SE (theta) } & \multirow[t]{2}{*}{ Marginal reliability } & \multicolumn{2}{|c|}{ Estimated theta } & \multirow[t]{2}{*}{$r$} \\
\hline & Mean & SD & & & & Mean & SD & \\
\hline None & 63 & 0 & 100 & 0.173 & 0.970 & -0.901 & 0.421 & 1 \\
\hline SE (theta) $\leq 0.447$ & 4.953 & 0.475 & 7.9 & 0.423 & 0.821 & -0.039 & 0.476 & $0.947^{\star \star}$ \\
\hline $\mathrm{SE}$ (theta) $\leq 0.386$ & 6.535 & 0.687 & 10.4 & 0.372 & 0.861 & -0.042 & 0.491 & $0.901^{* *}$ \\
\hline $\mathrm{SE}$ (theta) $\leq 0.316$ & 10.137 & 0.848 & 16.1 & 0.309 & 0.904 & -0.035 & 0.478 & $0.873^{\star \star}$ \\
\hline SE $($ theta) $\leq 0.224$ & 24.935 & 1.110 & 39.6 & 0.222 & 0.951 & -0.037 & 0.421 & $0.891^{* *}$ \\
\hline
\end{tabular}

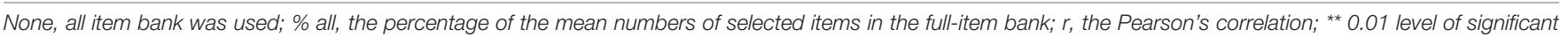
correlation (two-tailed).

Figure 1 reveals the findings of the test information function (TIF) and SEM across the theta under the simulated data of the CAT-ER. The greater the theta value, the greater the ER. More information denotes greater precision for measurement. A reliability coefficient of 0.85 or higher suggests that the measure has a good reliability (May et al., 2006). Naturally, the low-SEM criterion is a value of 0.39 or higher. These values are considered acceptable, because all the SEM values were under 0.39. The greater the TIF at each theta level, the smaller the SEM. According to the results, it is easy to see that the CAT-ER achieved adequate information and a reasonable standard error.

\section{Results Based on Real Data of the CAT-ER}

Table 7 displays the results of the real data for the CAT-ER under several stopping rules. The results were consistent with the aforementioned results of simulated-data CAT-ER simulation. Moreover, the number of selected items in real-data simulation was even lower than that in the simulated-data simulation. Furthermore, we depicted an additional graph (Figure 2) that visually indicated the number of selected items across the theta under different stopping rules, illustrating that most items executed were below the horizontal line labeled as 25 items, that is, $39.6 \%$ of entire item bank. Overall, all these findings demonstrate the CAT-ER performed well in the realdata simulation.

As shown in Table 8, the Pearson's correlations between the CAT-ER and the LOT-R varied from 0.813 to 0.851 under several stopping rules, and there was statistically significant difference $(p<0.01)$. This means that regardless of the stopping rule used, the CAT-ER had acceptable criterion-related validity.

\section{DISCUSSION}

In this study, we developed a CAT-ER as a novel and effective assessment of ER. Psychometric evaluations were implemented in the initial ER item bank, and items were excluded until all the remaining items had favorable psychometric properties. 


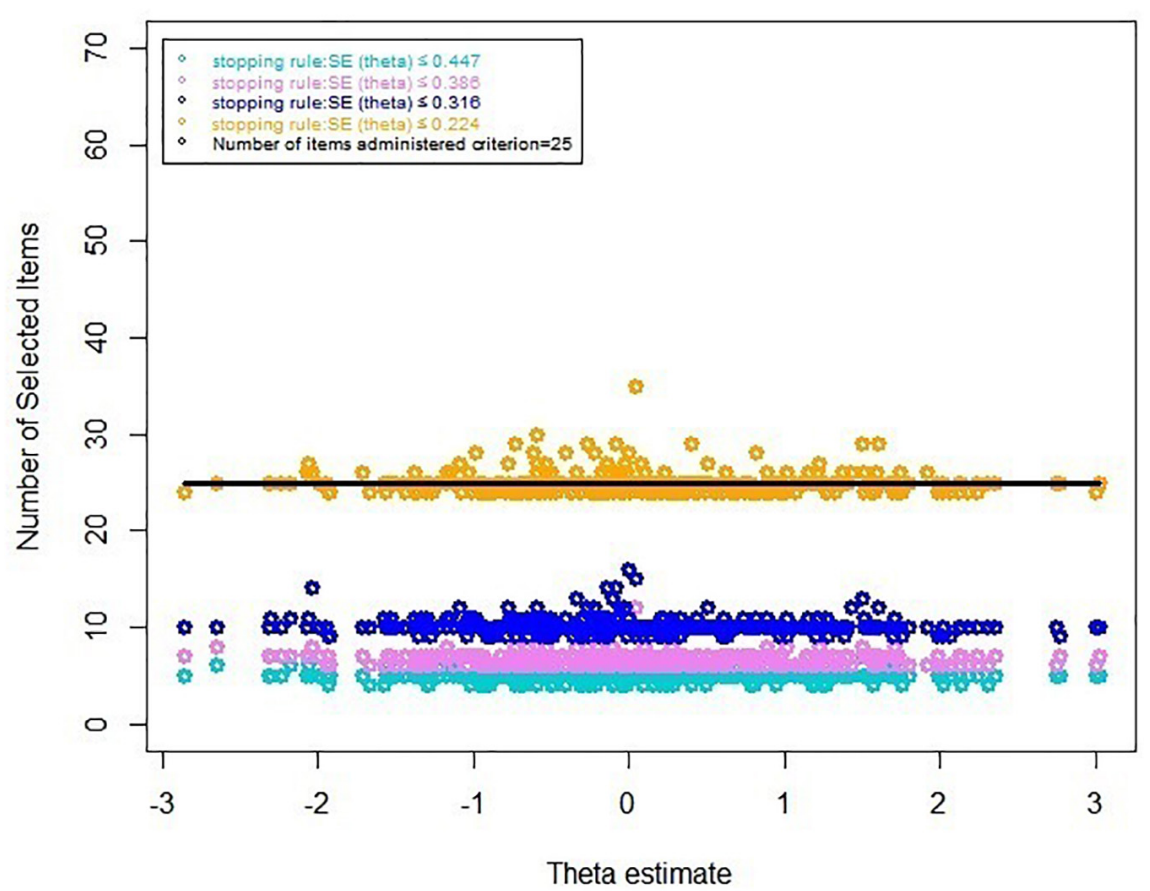

FIGURE 2 | Number of selected items under several stopping rules.

Subsequently, the final CAT-ER item bank (based on two simulation studies) was evaluated for efficiency, reliability, and validity. The findings indicated that the final 63 -item set satisfied the unidimensional and local independent assumptions, fitted the GRM well, had high item discrimination parameters, acceptable item fit, and no DIF. Furthermore, the proposed CAT-ER could greatly lessen the number of test items and thereby decrease the test burden on subjects, while also possessing desirable reliability and criterion-related validity. Specifically, there was no DIF based on gender, region, or age of subjects, which improves our confidence in advancing this CAT-ER version.

Researchers suggest that each measure mentioned above is formed based on the same underlying ER structure (Garnefski et al., 2001; Gross and John, 2003; Gratz and Roemer, 2004; Caprara et al., 2008). Furthermore, critical evidence from several studies on ER (Aldao et al., 2010; Gross, 2014; NaragonGainey et al., 2017; Megreya et al., 2018) indicates that each

TABLE 8 | Criterion-related validity of CAT-ER with external criteria scale under several stopping rules.

\begin{tabular}{lc}
\hline Stop rules & LOT-R \\
\hline None & $0.851^{* *}$ \\
SE (theta) $\leq 0.447$ & $0.813^{\star *}$ \\
SE (theta) $\leq 0.386$ & $0.827^{\star *}$ \\
SE (theta) $\leq 0.316$ & $0.829^{* *}$ \\
SE (theta) $\leq 0.224$ & $0.837^{\star *}$ \\
\hline None, all item bank was used; LOT-R, the revised Life Orientation Test; * 0.01 level \\
of significant correlation (two-tailed).
\end{tabular}

scale measures and evaluates the same underlying structure. Regarding empirical evidence, the one-factor CFA model and the dimensionality evaluation of IRT methods demonstrated that the final 63-item set of CAT-ER satisfied the hypothesis of unidimensionality. Consistently, a bifactor analysis suggested a dominant general ER factor that can be extracted in these ER items. The results further strengthen the usual practice of employing the total score of these measures as a general index of ER. The degree of correlations among test mean scores of these measures ranged from 0.66 to 0.83 , which indicates moderate to high correlations $(p<0.01)$, suggesting that they measured the underlying ER structure. Overall, both theoretical and empirical findings suggest the same underlying structure (i.e., ER) for the self-report ER measures in this study. Furthermore, studies indicated that ER is a rather complex construct; the inconsistencies with respect to the precise number and composition of the subscales are main obstacles to carrying out multidimensional scoring structures for ER measures (Catanzaro and Mearns, 1990; Salovey et al., 1995; Garnefski et al., 2001; Gross and John, 2003; Gratz and Roemer, 2004; Caprara et al., 2008). Fliege et al. (2005) studied CAT on depression levels (D-CAT) and Walter et al. (2007) on anxiety levels (AnxietyCAT), both single-dimensional CAT, even though depression and anxiety are both multidimensional. Based on the theoretical and empirical analyses, as well as common practices in previous studies, a unidimensional CAT-ER was developed to measure and assess the individual's overall ER in the present study.

Compared with previous studies, this study has some unique advantages. First, methodologically, this study used CAT to assess ER by establishing an effective item bank based on IRT 
methods, whereas questionnaires were used in most previous studies to evaluate ER based on CTT methods. IRT accounts for parameter invariance, which can ensure that the result will be unaffected by other results regardless of whether the subject is from a representative sample. Accordingly, the CAT-ER can evaluate the individual's ER precisely and effectively. Second, different test score systems can be compared in the CAT-ER. This approach ensures the difficulty of endorsing an item and the subjects' ER level to be on the same scale. Third, although many screening measures are available, the agreement between them is less than optimal, and no measure can be deemed a gold standard (Garnefski et al., 2001; Gross and John, 2003; Garnefski and Kraaij, 2006; Nadia and Vivian, 2007; Caprara et al., 2008). It accordingly might be difficult for researchers and clinicians to select an optimal instrument while evaluating ER. However, based on this study, it is suggested that subjects' ER can be estimated without the need to select a specific questionnaire.

This study demonstrated that the CAT-ER had acceptable efficiency, reliability, and validity. However, Figure 1 indicates that the CAT-ER offered little test information for those with a theta above 3 or below -3 , implying that the estimation precision of the CAT-ER for these subjects is uncertain. Future studies could develop a CAT suitable for these subjects. In addition, in a real-life setting, subjects' responses will be affected by many factors because the real-data simulation study may be different from a realistic environment (Smits et al., 2011). Based on the findings of a previous study (Kocalevent et al., 2009), the performance of CAT simulation was consistent with actual management findings, but it is uncertain that the similar outcome can be extended to an actual working CAT-ER program. Therefore, future studies could aim to design a CAT-ER for application in real-life settings.

Furthermore, several limitations should be considered in future studies. First, unidimensionality may not be fully satisfied in practice. For instance, many psychological and healthrelated tests are multidimensional measures. Multidimensional CAT or cognitive diagnostic CAT can be considered to treat multidimensional measures. Second, local dependency usually exists in some psychological or educational tests. The testlet model can be employed to address local dependency between items in future research. Third, the test developers still have much research to complete since the CAT procedure must be carefully conducted and maintained. Fourth, the maximum

\section{REFERENCES}

Akaike, H. T. (1974). A new look at the statistical model identification. IEEE Trans. Autom. Contr. 19, 716-723. doi: 10.1007/978-1-4612-1694-0_16

Aldao, A., Nolen-Hoeksema, S., and Schweizer, S. (2010). Emotion-regulation strategies across psychopathology: a meta-analytic review. Clin. Psychol. Rev. 30, 217-237. doi: 10.1016/j.cpr.2009.11.004

Balzarotti, S., John, O. P., and Gross, J. J. (2010). An Italian adaptation of the emotion regulation questionnaire. Eur. J. Psychol. Assess. 26, 61-67. doi: 10. 1027/1015-5759/a000009

Bock, R. D. (1972). Estimating item parameters and latent ability when responses are scored in two or more nominal categories. Psychometrika 37, 29-51. doi: 10.1007/BF02291411
Fisher information item selection rule was selected in this study due to its popularity (Magis and Barrada, 2017). Future research should choose various item selection rules, for example, the global information item selection rule, to enhance measurement precision.

\section{CONCLUSION}

The CAT-ER item bank had acceptable psychometric properties in the IRT and showed desirable performance in reducing the number of selected items without decreasing measurement accuracy. As a reliable and effective evaluation tool, the CATER can evaluate crucial clinical or practical problems and help promote the development of effective ER interventions.

\section{DATA AVAILABILITY STATEMENT}

The original contributions presented in the study are included in the article/supplementary material, further inquiries can be directed to the first author.

\section{ETHICS STATEMENT}

The studies involving human participants were reviewed and approved by the local Ethics Committee of School of Psychology, South China Normal University. The participants provided their written informed consent to participate in this study.

\section{AUTHOR CONTRIBUTIONS}

LX: data analysis and paper writing. RJ: data collection. FH and ZL: paper guidance. YZ: data collection. MZ: method guidance. All authors contributed to the article and approved the submitted version.

\section{FUNDING}

This research was supported by the Innovation Project of Graduate School of South China Normal University.

Bock, R. D., and Mislevy, R. J. (1982). Adaptive EAP estimation of ability in a microcomputer environment. Appl. Psychol. Measur. 6, 431-444. doi: 10.1177/ 014662168

Bornovalova, M. A., Gratz, K. L., Daughters, S. B., Nick, B., Delany-Brumsey, A., Lynch, T. R., et al. (2008). A multimodal assessment of the relationship between emotion dysregulation and borderline personality disorder among inner-city substance users in residential treatment. J. Psychiatr. Res. 42, 717-726. doi: 10.1016/j.jpsychires.2007.07.014

Campos, J. J., Walle, E. A., Dahl, A., and Main, A. (2011). Reconceptualizing emotion regulation. Emot. Rev. 3, 26-35. doi: 10.1177/17540739103 80975

Caprara, G. V., Di Giunta, L., Eisenberg, N., Gerbino, M., Pastorelli, C., and Tramontano, C. (2008). Assessing regulatory emotional self-efficacy 
in three countries. Psychol. Assess. 20, 227-237. doi: 10.1037/1040-3590. 20.3.227

Catanzaro, S. J., and Mearns, J. (1990). Measuring generalized expectancies for negative mood regulation: initial scale development and implications. J. Pers. Assess. 54, 546-563. doi: 10.1080/00223891.1990.9674019

Chalmers, R. P. (2012). Mirt: a multidimensional item response theory package for the R environment. J. Statist. Softw. 48, 1-29. doi: 10.18637/jss.v0 48.106

Chang, H. H., and Ying, Z. L. (1996). A global information approach to computerized adaptive testing. Appl. Psychol. Measur. 20, 213-229. doi: 10. $1177 / 0146621696$

Choi, S. W. (2015). Lordif: Logistic Ordinal Regression Differential Item Functioning Using IRT. Oxford: Oxford University Press.

Crane, P. K., Gibbons, L. E., Jolley, L., and van Belle, G. (2006). Differential item functioning analysis with ordinal logistic regression techniques: DIFdetect and difwithpar. Med. Care 44, S115-S123. doi: 10.1097/01.mlr.0000245183. 28384.ed

Finch, H., and Habing, B. (2007). Performance of dimtest- and noharm-based statistics for testing unidimensionality. Appl. Psychol. Measur. 31, 292-307. doi: $10.1177 / 0146621606294490$

Flens, G., Smits, N., Terwee, C. B., Dekker, J., Huijbrechts, I., and De Beurs, E. (2017). Development of a computer adaptive test for depression based on the dutch-flemish version of the promis item bank. Evaluat. Health Prof. 40, 79-105. doi: $10.1177 / 0163278716684168$

Fliege, H., Becker, J., Walter, O. B., Bjorner, J. B., Klapp, B. F., and Rose, M. (2005). Development of a computer-adaptive test for depression (D-CAT). Q. Life Res. 14, 2277-2291. doi: 10.1007/s11136-005-6651-9

Friedman, J., Hastie, T., and Tibshirani, R. (2010). Regularization paths for generalized linear models via coordinate descent. J. Stat. Softw. 33, 1-22. doi: 10.1016/j.jspi.2009.07.020

Gardner, W., Shear, K., Kelleher, K. J., Pajer, K. A., Mammen, O., Buysse, D., et al. (2004). Computerized adaptive measurement of depression: a simulation study. BMC Psychiatry 4:13. doi: 10.1186/1471-244x-4-13

Garnefski, N., and Kraaij, V. (2006). Relationships between cognitive emotion regulation strategies and depressive symptoms: a comparative study of five specific samples. Pers. Individ. Differ. 40, 1659-1669. doi: 10.1016/j.paid.2005. 12.009

Garnefski, N., Kraaij, V., and Spinhoven, P. (2001). Negative life events, cognitive emotion regulation and emotional problems. Pers. Individ. Diffe. 30, 1311-1327. doi: 10.1016/S0191-8869(00)00113-6

George, D., and Mallery, P. (2016). IBM SPSS Statistics 23 Step by Step: A Simple Guide and Reference. New York, NY: Routledge.

Gratz, K. L., and Roemer, L. (2004). Multidimensional assessment of emotion regulation and dysregulation: development, factor structure, and initial validation of the difficulties in emotion regulation scale. J. Psychopathol. Behav. Assess. 26, 41-54. doi: 10.1007/s10862-008-9102-4

Green, B. F., Bock, R. D., Humphreys, L. G., Linn, R. L., and Reckase, M. D. (1984) Technical guidelines for assessing computerized adaptive tests. J. Educ. Measur. 21, 347-360. doi: 10.1111/j.1745-3984.1984.tb01039.x

Gross, J. J. (ed.) (2014). Handbook of Emotion Regulation. New York, NY: Guilford publications.

Gross, J. J., and John, O. P. (2003). Individual differences in two emotion regulation processes: Implications for affect, relationships and well-being. J. Pers. Soc. Psychol. 85, 348-362. doi: 10.1037/0022-3514.85.2.348

$\mathrm{Gu}$, H. L., and Wang, C. K. (2012). Statistical control of the method effects associated with item wording:the chinese version of life orientation test-revised as an example. J. Psychol. Sci. 35, 1247-1253. doi: 10.16719/j.cnki.1671-6981. 2012.05.037

Gullone, E., and Taffe, J. (2011). The emotion regulation questionnaire for children and adolescents (ERQ-CA): a psychometric evaluation. Psychol. Assess. 24 409-417. doi: 10.1037/a0025777

Kang, T., and Chen, T. T. (2008). Performance of the generalized S-X2 item fit index for polytomous IRT models. J. Educ. Measur. 45, 391-406. doi: 10.2307/ 20461906

Kline, R. B. (2010). Principles and Practice of Structural Equation Modeling New York, NY: Guilford Press.

Kocalevent, R.-D., Rose, M., Becker, J., Walter, O. B., Fliege, H., Bjorner, J. B. et al. (2009). An evaluation of patient-reported outcomes found computerized adaptive testing was efficient in assessing stress perception. J. Clin. Epidemiol. 62, 278-287.e3. doi: 10.1016/j.jclinepi.2008.03.003

Lavender, J. M., Wonderlich, S. A., Peterson, C. B., Crosby, R. D., Engel, S. G., Mitchell, J. E., et al. (2014). Dimensions of emotion dysregulation in bulimia nervosa. Eur. Eat. Disord. Rev. 22, 212-216. doi: 10.1002/erv. 2288

Li, C. H., and Wu, J. J. (2018). Psychometric evaluation of the chinese version of the emotion regulation questionnaire in taiwanese college students. Assessment 27, 1300-1309. doi: 10.1177/1073191118773875

Li, C. Y., Yan, J., Yin, X. P., and Wu, Z. Y. (2002). Application of the trait metamood scale (tmms) in military students. Chin. J. Clin. Psychol. 10, 202-203. doi: 10.3969/j.issn.1005-3611.2002.03.014

Linden, W. J. V. D. (1998). Bayesian item selection criteria for adaptive testing. Psychometrika 63, 201-216. doi: 10.1007/BF02294775

Magis, D., and Barrada, J. R. (2017). Computerized adaptive testing with R: recent updates of the package catR. J. Statist. Softw. 76, 1-19. doi: 10.18637/jss.v0 76.c01

Magis, D., and Raiche, G. (2012). Random generation of response patterns under computerized adaptive testing with the R package catR. J. Statist. Softw. 48, 1-31. doi: 10.18637/jss.v048.i08

May, S., Littlewood, C., and Bishop, A. (2006). Reliability of procedures used in the physical examination of non-specific low back pain: a systematic review. Austr. J. Physiother. 52, 91-102. doi: 10.1016/s0004-9514(06)70044-7

Maydeu-Olivares, A., and Joe, H. (2006). Limited information goodness-of-fit testing in multidimensional contingency tables. Psychometrika 71, 713-732. doi: 10.1007/s11336-005-1295-9

Megreya, A. M., Latzman, R. D., Al-Emadi, A. A., and Al-Attiyah, A. A. (2018). An integrative model of emotion regulation and associations with positive and negative affectivity across four Arabic speaking countries and the USA. Motiv. Emot. 42, 566-575. doi: 10.1007/s11031-018-9682-6

Mennin, D. S., McLaughlin, K. A., and Flanagan, T. J. (2009). Emotion regulation deficits in generalized anxiety disorder, social anxiety disorder, and their co-occurrence. J. Anxiety Disord. 23, 866-871. doi: 10.1016/j.janxdis.2009. 04.006

Muraki, E. (1992). A generalized partial credit model: application of an EM algorithm. Appl. Psychol. Measur. 16, 159-176. doi: 10.1002/j.2333-8504.1992. tb014.36.x

Muthén, L. K., and Muthén, B. O. (2012). Mplus Version 7 User's Guide. Los Angeles, CA: Muthén and Muthén.

Nadia, G., and Vivian, K. (2007). The cognitive emotion regulation questionnaire. Eur. J. Psychol. Assess. 23, 141-149. doi: 10.1027/1015-5759.23. 3.141

Naragon-Gainey, K., Mcmahon, T. P., and Chacko, T. P. (2017). The structure of common emotion regulation strategies: a meta-analytic examination. Psychol. Bull. 143, 384-427. doi: 10.1037/bul0000093

Reise, S. P., Morizot, J., and Hays, R. D. (2007). The role of the bifactor model in resolving dimensionality issues in health outcomes measures. Q. Life Res. 16, 19-31. doi: 10.1007/s11136-007-9183-7

Reise, S. P., Scheines, R., Widaman, K. F., and Haviland, M. G. (2013). Multidimensionality and structural coefficient bias in structural equation modeling: a bifactor perspective. Educ. Psychol. Measur. 73, 5-26. doi: 10.1177/ 0013164412449831

Reise, S. P., and Yu, J. (1990). Parameter recovery in the graded response model using multilog. J. Educ. Measur. 27, 133-144. doi: 10.1111/j.1745-3984.1990. tb00738.x

Salovey, P., Mayer, J. D., Goldman, S. L., Turvey, C., and Palfai, T. P. (1995). "Emotional attention, clarity, and repair: exploring emotional intelligence using the trait meta-mood scale," in Emotion, Disclosure, and Health, ed. J. W. Pennebaker (Washington, DC: American Psychological Association), 125-154. doi: $10.1037 / 10182-006$

Samejima, F. (1969). Estimation of latent ability using a response pattern of graded scores. Psychometr. Monogr. 17, 5-17. doi: 10.1007/BF033 72160

Scheier, M. F., and Carver, C. S. (1985). Optimism, coping, and health: assessment and implications of generalized outcome expectancies. Health Psychol. 4, 219 247. doi: 10.1037//0278-6133.4.3.219

Scheier, M. F., Carver, C. S., and Bridges, M. W. (1995). Distinguishing optimism from neuroticism (and trait anxiety, self-mastery, and self-esteem) 
a reevaluation of the life orientation test. J. Pers. Soc. Psychol. 67, 1063-1078. doi: 10.1037//0022-3514.67.6.1063

Schwarz, G. E. (1978). Estimating the dimension of a model. Ann. Statist. 6, 461-464. doi: 10.1214/aos/1176344136

Smits, N., Cuijpers, P., and van Straten, A. (2011). Applying computerized adaptive testing to the CES-D scale: a simulation study. Psychiatry Res. 188, 147-155. doi: 10.1016/j.psychres.2010.12.001

Spiegelhalter, D. J., Best, N. G., and Carlin, B. P. (1998). Bayesian deviance, the effective number of parameters, and the comparison of arbitrarily complex models. Res. Rep. 64, 98-99.

Wainer, H., Bradlow, E. T., and Du, Z. (2000). "Testlet response theory: an analog for the 3PL model useful in testlet-based adaptive testing," in Computerized Adaptive Testing: Theory and Practice, eds W. J. van der Linden and G. A. W. Cees (Dordrecht: Kluwer Academic Publishers), 245-269. doi: 10.1007/0-30647531-6_13

Walter, O. B., Becker, J., Bjorner, J. B., Fliege, H., Klapp, B. F., and Rose, M. (2007). Development and evaluation of a computer adaptive test for 'Anxiety' (Anxiety-CAT). Q. Life Res. 16, 143-155. doi: 10.1007/s11136-007-9191-7

Wang, G., Mearns, J., Yang, X., Han, P., and Catanzaro, S. J. (2017). Measuring generalised expectancies for negative mood regulation in china: the chinese language negative mood regulation scale. Intern. J. Psychol. 54, 223-231. doi: 10.1002/ijop.12443
Wen, S., Tang, D., and Yu, G. (2009). The characteristics of regulatory emotional self-efficacy in Chinese graduate students. Psychol. Sci. 32, 666-668. doi: 10. 16719/j.cnki.1671

Wickham, H. (2011). ggplot2. WIREs Computat. Statist. 3, 180-185. doi: 10.1002/ wics. 147

Yen, W. M. (1993). Scaling performance assessments: strategies for managing local item dependence. J. Educ. Measur. 30, 187-213. doi: 10.1111/j.1745-3984.1993. tb00423.x

Zhu, X., Auerbach, R. P., Yao, S., Abela, J. R. Z., Xiao, J., and Tong, X. (2008). Psychometric properties of the cognitive emotion regulation questionnaire: chinese version. Cogn. Emot. 22, 288-307. doi: 10.1080/02699930701369035

Conflict of Interest: The authors declare that the research was conducted in the absence of any commercial or financial relationships that could be construed as a potential conflict of interest.

Copyright (C) $2020 \mathrm{Xu}$, Jin, Huang, Zhou, Li and Zhang. This is an open-access article distributed under the terms of the Creative Commons Attribution License (CC BY). The use, distribution or reproduction in other forums is permitted, provided the original author(s) and the copyright owner(s) are credited and that the original publication in this journal is cited, in accordance with accepted academic practice. No use, distribution or reproduction is permitted which does not comply with these terms. 